\title{
SYNTHESIS OF EXOSKELETON CONTROL ALGORITHMS BASED ON KINEMATIC ANALYSIS OF LOCOMOTIONS AND HUMAN GAIT MODELLING
}

\author{
Yury Loskutov' ${ }^{1}$, Alexandr Kapustin ${ }^{1}$, Alexandr Kudryavtsev' ${ }^{1}$, Albert Nasibullin ${ }^{2}$, Alexandra Lebedeva ${ }^{3}$ \\ ${ }^{1}$ Volga State University of Technology, Russian Federation \\ 2Joint stock company "Volzhsky Electromechanical Factory" \\ 3Joint stock company "Semiconductor device plant"
}

The article considers a mathematical model of human gait. Gait parameters necessary to build an analytical model of motion are defined. Calculated ratio to define the coordinates of key points of legs at any moment of time is obtained and a kinematic analysis of locomotions is performed. The results of computer modelling of walking process are given. The calculated ratio and the mathematical model can be used for design process and programming of the operation of control systems of exoskeleton developed for medical purposes.

Key words: Exoskeleton; Human locomotion; Mathematical modelling; Computer simulation; Synthesis and analysis of mechanisms

\section{INTRODUCTION}

The last few decades $[1,2]$ have seen an active investigation into the development of the exoskeleton. An exo-

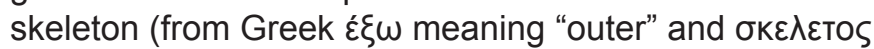
meaning "skeleton") is a device designed to increase human strength due to the outer framework (namely, an exoskeleton or a suit). This device can be used not only to increase the strength of an able-bodied person but also to assist people suffering from musculoskeletal disorders (Figure 1) [3].

The basic elements of exoskeletons are a power source, a programmable control system and a mechanical exoskeleton with drives. It should be noted that problems concerning the design and improvement of each of these elements are equally important.

When designing the control system it is necessary to
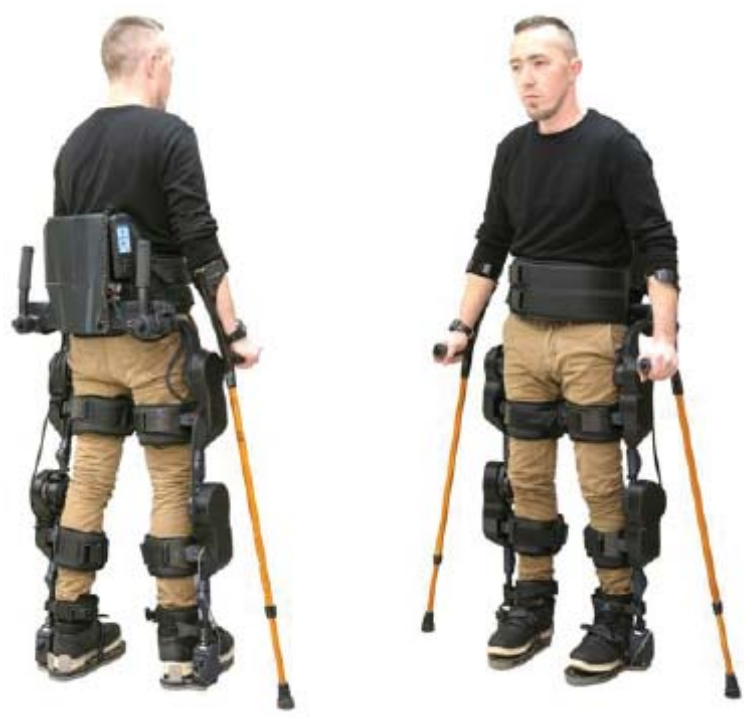

know the laws of motion [4] to eliminate extra and unnecessary movements. The response time of exoskeleton elements is also essential [5]. Asynchronous behaviour can cause serious health problems. An exoskeleton must "feel" or even "predict" the movements of a person [6]. To fix and process random or unnecessary movements, an exoskeleton must include suitable sensors [7] which would respond to the actions and position of a body in space taking into account correct locomotions and conditions for the balance stability of a person.

That is why the modelling of the correct locomotions of an able-bodied person is necessary to generate the laws of motion and the correlation of exoskeleton movements with a patient are still considered to be important.

The aim of the research work is to develop a mathematical model for a human gait and synthesize the algorithms for exoskeleton control.

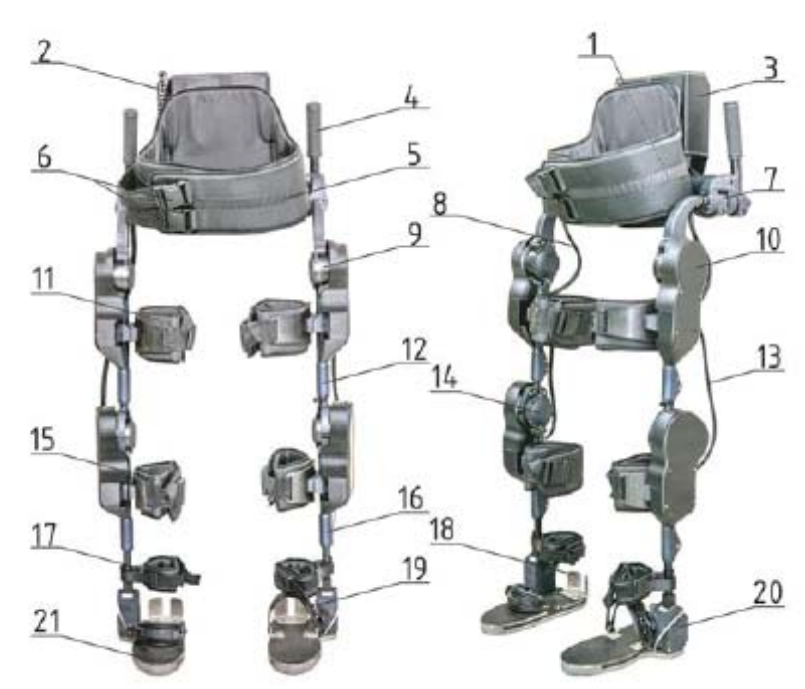

Figure 1 
The tasks to be solved:

1. development of the computed mathematical model of human gait;

2. kinematic analysis of locomotion while walking;

3. computer simulation of the walking process;

4. synthesis of the laws of motion for the exoskeleton servodrive control system.

\section{DESCRIPTION OF THE DEVELOPED MODEL}

Figure 1 shows a prototype of an exoskeleton. The construction includes the following elements: 1 - adjustable waist unit; 2 - controller; 3 - power supply system; 4 - support handle; 5 - fixing system for a lumbar part; 6 - fastex (plas-tic fastener) to regulate the pelvic belt; 7 - stop; 8 - upper flexible cable; 9 - electric drive of a thigh joint; 10 - protective cover; 11 - thigh support; 12 - adjustment unit of the thigh length; 13 - lower flexible cable; 14 - electric drive of the knee joint; 15 and 17 - shank supports; 16 - adjustment unit of the shank length; 18 - heel fixing element; 19 - foot attachment clip; 20 - elastic joint; 21 - sole with sensors.

An important problem when designing an exoskeleton is the choice of a power drive unit. For a rehabilitation exoskeleton for medical purposes (REM) [8], we have chosen a rather light-weight, easily programmable and stable for motion processing electric servo drive. The designed drive includes electric motors, cycloidal gear reducers and feedback sensors. The servo drive must provide a complete range of set movements (angles and angular velocities), consume small amounts of energy and have a small size and weight.

\section{DESIGN MODEL}

Walking is the most natural way of human motion. It is an automated motor action produced as a result of a complex coordinate activity of skeletal muscles of a body and limbs $[9,10]$. The basic functional unit of a gait is a gait cycle, i.e. time from the moment when the foot touches the ground to the next touch with the same foot [9]. The gait cycle is measured in seconds. Other time features, as a rule, are measured in relative units, e.g. percentage of step cycle time of a given side.

There can be distinguished right and left gait cycles. The gait cycle for a leg consists of a stance phase and a swing phase (Figure 2, [11]). The length of the stance phase varies between 58 and $61 \%$ of a gait cycle, and the length of the swing phase is between 42 and $39 \%$ of the gait cycle. Due to the fact that both legs are involved into walking process, the stance phase part is the time when both legs touch the ground. This time is called a double support phase and constitutes $16-22 \%$ of a gait cycle. Double support occurs twice - at the beginning and at the end of the stance phase. Each period of a double support phase ranges from 8 to $11 \%$ of the gait cycle length. The swing phase of a behind-standing leg for the support leg launches the single support phase. The single support phase is equal to the corresponding swing phase of the other leg.

The transfer from the walking process of a human to walking process of an exoskeleton is a task of physical mathematical modelling of the sequence of regular movements of human bones and joints. The kinematic system of the human skeleton is a complex mechanical system which has a lot of degrees of freedom. Mathematical modelling of such a system is very difficult due to the excessive multifractality of the model. Therefore, to make up a working design model it is necessary to introduce a series of assumptions and changes. Taking into ac-count the previous experience of the scientists working in the field of biomechanics [3-7, 9-12], theoretical mechanics and the theory of mechanisms and machines, as well as drawing on the technical project to design and develop the high-tech production of a multifunctional ro-botic exoskeleton for medical purposes, we can accept the following kinematic scheme of a human walking mechanism (see Figure 3). While drawing a mathematical model, we introduced the following assumptions and simplifications:

- only the lower part of the human skeleton (pelvic bones) which participate in the walking process as a

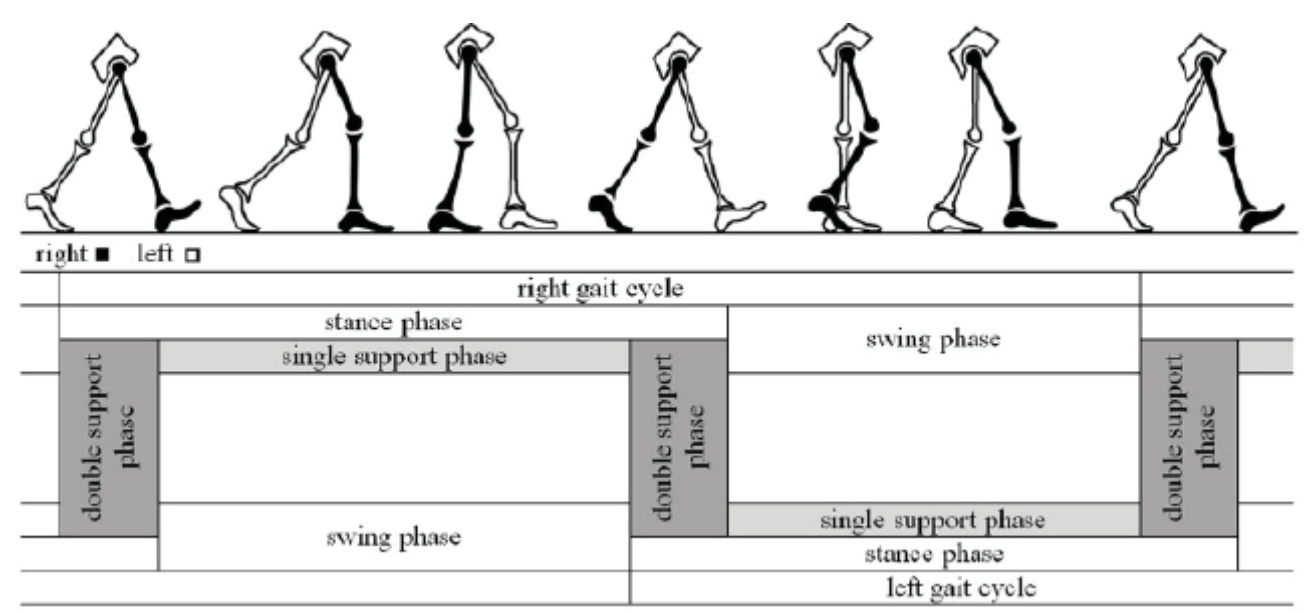

Figure 2: Gait cycle components [11] 

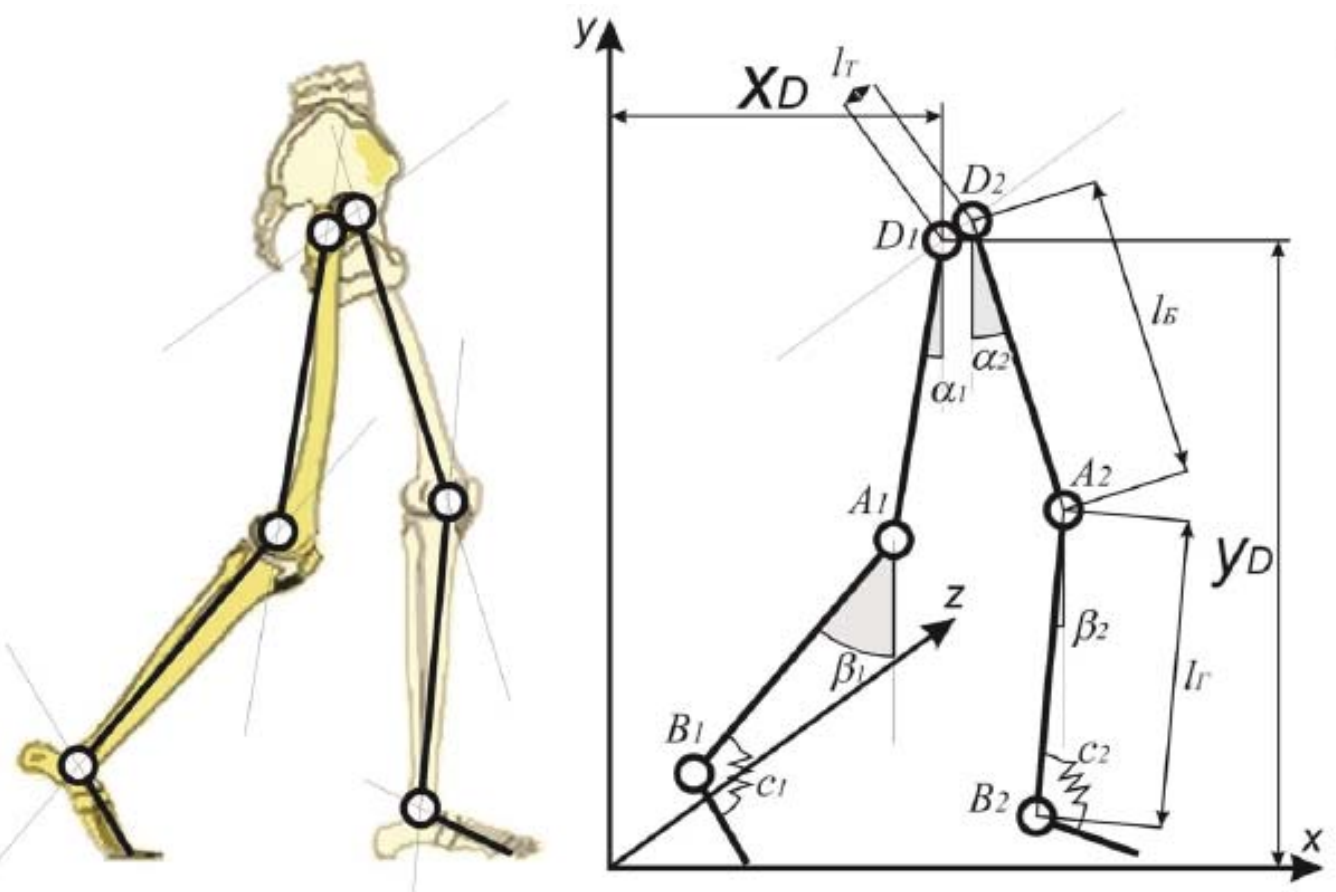

Figure 3: Walking phases and kinematic scheme of human gait mechanism

mover is considered;

- human bones are links in the kinematic chain and consequently are considered as solid bodies;

- a shinbone and a calf bone are changed into one link having the length of $\mathrm{I} \Gamma$;

- the thigh joint (D) which is a spherical kinematic pair (KP) and joints of the foot (B1 or B2) which are also a multi element spherical kinematic pair are changed into rotating KP. The rotation plane of these rotating $\mathrm{KPs}$ is in the sagittal plane during the walking process;

- we neglect the friction in the joints due to its low amount and consider the joints of the mechanism to be ideal;

- the foot is the link which does not have finger bends. It is connected to the shank joint II by means of flexible coupling;

- the complex form of the bones is changed into straight links connected by kinematic pairs;

- muscle which are one-way prismatic drives are changed into rotating drives in the ki-nematic pairs $D, A_{1}, A_{2}, B_{1}, B_{2}$. Master angles from the vertical axis are marked as $\alpha_{1}, \alpha_{2}, \beta_{1}, \beta_{2}$.

The computer kinematic scheme is a doubled flat open kinematic circuit which has 6 de-grees of freedom. To control the movement we will use controlling rotating drives in points $D, A_{1}, A_{2}$. In points $B_{1}, B_{2}$ there are flexible couplings which have the rigidity $C_{1}, C_{2}$.

\section{MATHEMATICAL MODELLING}

Walking in the forward direction is a cyclic process in which over one cycle a conditional point $B$ (human foot) makes a curve in coordinates $x, y$ relating to the ground. In this case point $D$ of the leg suspension (pelvic joint) also moves depending on the laws of angle changes $\alpha_{1}$, $\alpha_{2}, \beta_{1}, \beta_{2}$. Using the scheme given in Figure 3 we can

$$
\left\{\begin{array}{l}
x_{D}=l_{5} \sin \alpha+l_{\Gamma} \sin \beta+x_{B} \\
y_{D}=l_{5} \cos \alpha+l_{\Gamma} \cos \beta+y_{B}
\end{array}\right.
$$

represent this dependence as a following formula (1).

This dependence can be convenient for the description of the path described by a point $D$ which can be approximated by a periodic function in the direction of walking [9] and also represent as a suspension point for other locomotive motions of legs.

When people or animals walk the centre of gravity changes its position. For example, for a human, the centre of gravity and connected with it point $D$ of the leg suspension move in vertical plane forming a curve which is close to harmonic function (sinusoid or cosine curve) with a period which is equal to a half of the gait cycle length [9].

The modelling of a double-leg gait, except for ratio (1), should include the dependences which set the human body forward motion. Let us assume that the length of each step lw and length of the cycle $T$ are equal. Then, the human speed in the set mode will be constant and will equal $v$. The dependence of the coordinates of point $\mathrm{D}$ can be represented in the following way:

$$
\left\{\begin{array}{l}
x_{D}=\int \mathrm{v} d t \\
y_{D}=l_{5}+l_{\Gamma}+\frac{f_{D}}{2}\left[\cos \left(\frac{2 \pi}{0,5 T} t+\psi\right)-1\right]
\end{array}\right.
$$


where $T=\frac{2 l_{u}}{\mathrm{v}}=\frac{2 l_{u}}{\dot{x}_{D}}$ is gait cycle length which varies between 1.1 and 1.2 seconds at the medium walking rhythm $n ; f_{D}$ is a peak-to-peak swing of the human gravity centre fluctuations along the vertical axis; $\psi$ is the initial phase of fluctuations. At a medium rhythm of 100110 steps per minute and step length equal to $I_{m}=0,66 \div$ 0,7 meters, a peak-to-peak swing $f_{D}$ (which is equal to two amplitudes) can reach $4 \div 5 \mathrm{~cm}$ [9]. It is obvious that $y_{D}^{\max }=l_{\bar{B}}+l_{\Gamma}=f_{D}+y_{D}^{\min }$.

If the person moves steadily along the horizontal plane, the speed and acceleration pro-jections of the pelvic joint $D$ will equal to $(3,4)$.

If a straight-line motion is accelerated or slowed down,

$\left\{\begin{array}{l}x_{D}=\mathrm{v} \\ \dot{y}_{D}=-\frac{\pi \cdot f_{D}}{T} \sin \left(\frac{2 \pi}{T} t+\psi\right),\end{array}\right.$
$\left\{\begin{array}{l}\ddot{x}_{D}=0 \\ \ddot{y}_{D}=-\frac{2 \pi^{2} f_{D}}{T^{2}} \cos \left(\frac{2 \pi}{T} t+\psi\right) .\end{array}\right.$

$\left\{\begin{array}{l}\dot{x}_{D}=\mathrm{v} \\ \dot{y}_{D}=-\frac{f_{D} \pi}{l_{u \iota}}(\mathrm{v}+\dot{\mathrm{v}} \cdot t) \sin \left(\frac{2 \pi}{l_{u}} \mathrm{v} \cdot t+\psi\right)\end{array}\right.$

$\left\{\begin{array}{l}\ddot{x}_{D}=\dot{\mathrm{v}} \\ \ddot{y}_{D}=\dot{y}_{D}=-\frac{f_{D} \pi}{l_{u}}(2 \dot{\mathrm{v}}+\ddot{\mathrm{v}} \cdot t) \sin \left(\frac{2 \pi}{l_{u}} \mathrm{v} \cdot t+\psi\right)-\frac{2 f_{D} \pi^{2}}{l_{u u}{ }^{2}}(\mathrm{v}+\dot{\mathrm{v}} \cdot t)^{2} \cos \left(\frac{2 \pi}{l_{u}} \mathrm{v} \cdot t+\psi\right) .\end{array}\right.$

$\left\{\begin{array}{l}x_{A}=x_{D}+l_{5} \sin \alpha \\ y_{A}=y_{D}-l_{B} \cos \alpha\end{array}\right.$

$\left\{\dot{x}_{A}=\dot{x}_{D}+\dot{\alpha} \cdot l_{B} \cdot \cos \alpha\right.$

$\left\{\dot{y}_{A}=\dot{y}_{D}+\dot{\alpha} \cdot l_{B} \cdot \sin \alpha\right.$

$\left\{\ddot{x}_{A}=\ddot{x}_{D}+\ddot{\alpha} \cdot l_{B} \cdot \cos \alpha-(\dot{\alpha})^{2} \cdot l_{B} \cdot \sin \alpha\right.$

$\left\{\ddot{y}_{A}=\ddot{y}_{D}+\ddot{\alpha} \cdot l_{B} \cdot \sin \alpha+(\dot{\alpha})^{2} \cdot l_{D} \cdot \cos \alpha\right.$.

$\left\{x_{B}=x_{A}-l_{\Gamma} \sin \beta\right.$

$\left\{y_{B}=y_{A}-l_{\Gamma} \cos \beta\right.$

$\left\{\dot{x}_{B}=\dot{x}_{A}-\dot{\beta} \cdot l_{\Gamma} \cdot \cos \beta\right.$

$\left\{\dot{y}_{B}=\dot{y}_{A}+\dot{\beta} \cdot l_{\Gamma} \cdot \sin \beta\right.$

$\left\{\begin{array}{l}\ddot{x}_{B}=\ddot{x}_{A}-\ddot{\beta} \cdot l_{\Gamma} \cdot \cos \beta+(\dot{\beta})^{2} \cdot l_{\Gamma} \cdot \sin \beta \\ \ddot{y}_{B}=\ddot{y}_{A}+\ddot{\beta} \cdot l_{\Gamma} \cdot \sin \beta+(\dot{\beta})^{2} \cdot l_{\Gamma} \cdot \cos \beta .\end{array}\right.$

$\mathrm{v}_{A}=\sqrt{\dot{x}_{A}^{2}+\dot{y}_{A}^{2}}, \quad a_{A}=\sqrt{\ddot{x}_{A}^{2}+\ddot{y}_{A}^{2}}$,

$\mathrm{v}_{B}=\sqrt{\dot{x}_{B}^{2}+\dot{y}_{B}^{2}}, \quad a_{B}=\sqrt{\ddot{x}_{B}^{2}+\ddot{y}_{B}^{2}}$

$$
\left\{\begin{array}{l}
x_{\text {umb }}=x_{D}+0,44 l_{5} \sin \alpha \\
y_{\text {um }}=y_{D}-0,44 l_{5} \cos \alpha
\end{array}\right.
$$

$\left\{\dot{y}_{\text {um }}=\dot{y}_{D}+0,44 \dot{\alpha} \cdot l_{5} \cdot \sin \alpha\right.$

$\left\{\begin{array}{l}x_{u m \Gamma}=x_{A}-0,42 l_{\Gamma} \sin \beta \\ y_{\text {uym }}=y_{A}-0,42 l_{\Gamma} \cos \beta\end{array}\right.$

$\left\{\begin{array}{l}\dot{x}_{u m \Gamma}=\dot{x}_{A}-0,42 \dot{\beta} \cdot l_{\Gamma} \cdot \cos \beta \\ \dot{y}_{u m \Gamma}=\dot{y}_{A}+0,42 \dot{\beta} \cdot l_{\Gamma} \cdot \sin \beta\end{array}\right.$ in this case taking into account $T=\frac{2 l_{u}}{\mathrm{v}}=\frac{2 l_{u}}{\dot{x}_{D}}$, for

speed and acceleration projections we obtain $D(5)$.

Coordinate, speed and acceleration projections of a knee A $(6,7,8)$.

Coordinate, speed and acceleration projections of an ankle joint $B(9,10,11)$.

Absolute speed, acceleration points $A$ and $B$ at the considered moment of time will be equal to (12).

In case of dynamic calculations the necessary coordinates, speed and accelerations values of the gravity centers of the thigh and shank can be also defined in the same manner. Taking into account given in some works [13] averaged data of gravity centers of the thigh (located

$$
\left\{\begin{array}{l}
\ddot{x}_{u m \Gamma}=\ddot{x}_{A}-0,42 \ddot{\beta} \cdot l_{\Gamma} \cdot \cos \beta+0,42(\dot{\beta})^{2} \cdot l_{\Gamma} \cdot \sin \beta \\
\ddot{y}_{u m \Gamma}=\ddot{y}_{A}+0,42 \ddot{\beta} \cdot l_{\Gamma} \cdot \sin \beta+0,42(\dot{\beta})^{2} \cdot l_{\Gamma} \cdot \cos \beta
\end{array}\right.
$$




$$
\begin{aligned}
& \mathrm{v}_{u m b}=\sqrt{\dot{x}_{u m D^{2}}+\dot{y}_{u m b}{ }^{2}}, \quad a_{u m b}=\sqrt{\ddot{x}_{u m D^{2}+\ddot{y}_{u m}{ }^{2}}}, \\
& \mathrm{v}_{u m \Gamma}=\sqrt{\dot{x}_{u m \Gamma}{ }^{2}+\dot{y}_{u m \Gamma^{2}}}, \quad a_{u m \Gamma}=\sqrt{\ddot{x}_{u m \Gamma}{ }^{2}+\ddot{y}_{u m \Gamma} \Gamma^{2}} .
\end{aligned}
$$

at the distance of $0.44 \mathrm{I} 5$ from the pelvic joint) and of the shank (located at the distance of $0.42 \mathrm{I} \Gamma$ from the knee joint), we obtain $(13,14,15,16,17,18)$.

Finally, velocities and accelerations of the gravity centers of the thigh and shank are equal to $(19,20)$.

The given dependences are more suitable for revised design where the laws of angle changes in time will give the path of $D$ point of leg suspension, of $A$ point of knee joint and $B$ point of ankle joint.

To describe the process of movement we need inverse dependences where we can define angular values according to the set coordinates and geometrical parameters. Such dependences were developed and intro- duced, see [9-11, 14-16].

Let us consider one of the kinematic chains where the links are represented in the form of the closed paths of the vectors with the total vector $r_{0}$ (Figure 4). The angle between the links in the knee joint $\gamma=(\pi-\alpha-\beta)$ (Figure 4) defines the angle between the thigh and the knee. The work of the knee and thigh joints is described in works [9, 18]. The average mobility of the knee joint is within the range of $0^{\circ}-130^{\circ}$ (when the initial value equals $y=180^{\circ}$ ).

We have (21): $\alpha=\operatorname{arctg}\left(k_{1}\right)-\operatorname{arctg}\left(k_{2}\right)$

$$
\beta=\operatorname{arctg}\left(k_{1}\right)-\operatorname{arctg}\left(k_{2}\right)
$$

The values of angular velocities and accelerations are equal to (22).

$$
\begin{aligned}
& \operatorname{tg}\left(k_{1}\right)=\frac{\Delta_{x} \Delta_{y}+\sqrt{e^{2}\left(r_{0}^{2}-e^{2}\right)}}{\Delta_{z}^{2}-e^{2}}, \operatorname{tg}\left(k_{2}\right)=\sqrt{\frac{4\left(l_{B}+l_{\Gamma}\right)-r_{0}^{2}}{r_{0}^{2}-4\left(l_{B}+l_{\Gamma}\right)}}, \\
& \Delta_{x}=x_{D}-x_{B} ; \quad \Delta_{y}=y_{D}-y_{B} ; \quad e=\left(l_{5}-l_{\Gamma}\right) \sqrt{\frac{\left(l_{B}+l_{\Gamma}\right)^{2}-r_{0}^{2}}{l_{B} l_{\Gamma}}} ; \quad r_{0}^{2}=\Delta_{x}^{2}+\Delta_{y}^{2} . \\
& \ddot{\beta}=\frac{\ddot{x}_{A}-\ddot{x}_{B}+(\dot{\beta})^{2} \cdot l_{\Gamma} \cdot \sin \beta}{l_{\Gamma} \cdot \cos \beta}=\frac{\ddot{y}_{B}-\ddot{y}_{A}-(\dot{\beta})^{2} \cdot l_{\Gamma} \cdot \cos \beta}{l_{\Gamma} \cdot \sin \beta} \text {. }
\end{aligned}
$$

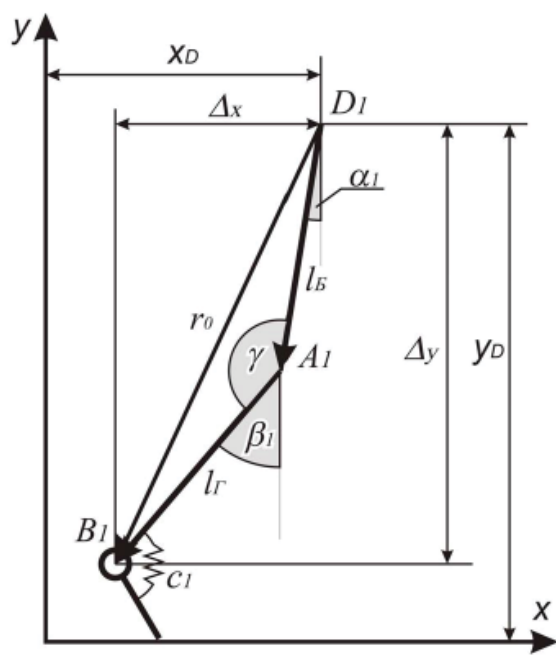

Figure 4: Kinematic chain 


\section{ALGORITHM OF VISUALIZATION AND COMPUTER MODELLING OF THE GAIT}

Based on the given above formulae [17] for walking, we introduced the algorithm of visualization (Figure 5).

The work [9] presents the results of the modelling of the common human gait having the following parameters: mass of the body is $56.7 \mathrm{~kg}$, thigh length is $0.314 \mathrm{~m}$, shank length is $0.425 \mathrm{~m}$. The gait cycle is divided into 70 positions. Based on the polynomials, we derived approximate angle functions (in degrees) for gait cycle length $\mathrm{T}$, where $0<\mathrm{t}<\mathrm{T}$. (24)

Figure 6 shows the diagrams of absolute angle changes for left and right legs based on the measurement data [9] and on the ratios (24). Figure 7 shows vertical coordinate changes in key points (heels, knee, hip joints) in time which are based on data [9] and ratios $(2,6,9)$.

Let us consider the motion of elements during the walking process based on [9-11]. In the hip joint during the gait cycle there is only one flexion and extension. Flexion amplitude at the medium gait rate is approximately 30 degrees, while that of the extension is 10 degrees.

The knee joint during the gait cycle makes four basic motions - two flexions and two ex-tensions. The first flexion starts at the end of the swing phase in $96-97 \%$ of the cycle and con-tinues up to the beginning of the single support phase. Its amplitude is an average 15 degrees. Then the flexion turns into gradual extension. When the next double support phase begins, the second flexion occurs. It is fast and high-amplitude (60-70 degrees). It finishes in the middle of the swing phase. After that? the joint quickly unbends.
Ankle joint in the exoskeleton has an elastic joint with shank element.

One can use different approaches to model the walking process of a human, and the final results can be the angular functions as well as other options. The obtained dependences of the angular values can be input into the microcontroller which controls the electric rotary drives in pelvic and knee joints. For different locomotions, according to the models, we can calculate functional dependences, including those for transient motions [4, 5, 7-10]. Computer modelling of the locomotion process is considered to be very important at this stage of design.

The link https://alexanderfadeev.github.io/legmovement. $\mathrm{htm} /$ provides an access to the software realization with WEB interface of the computer modelling of the locomotions while walking (Figure 8). The visualization is based on the described above mathematical model of the flat kinematic chain of the human legs. The program set the following parameters: $I_{5}$ - thigh length, $I_{\Gamma}$ - shank length; $\alpha_{0}$ and $\beta_{0}$ - initial values of angles; $\alpha=f_{1,2}(t)$ and $\beta=f_{1,2}(t)$ - time functions of angles for moving (1) and supporting (2) legs; parameters for inverting and in-vestigation of functions. The block-diagram of the software operation is given in Figure 5.

The application of laws of angle changes (24) for a cycle 0.987 seconds long gives a rather adequate gait model.

The analysis of the paths of the key points and visualization of the locomotions of a hu-man being while walking can point to the conclusion about the reliability of the developed mathematical and computer models.

$$
\begin{aligned}
& \alpha_{s}=5915.1 \cdot t^{6}-14483 \cdot t^{5}+11532 \cdot t^{4}-2992.6 \cdot t^{3}+117.38 \cdot t^{2}-78.151 \cdot t+17.399, \\
& \alpha_{2}=14.585 \cdot t^{6}+1286.9 \cdot t^{5}-3148.2 \cdot t^{4}+3020 \cdot t^{3}-1547.7 \cdot t^{2}+381.35 \cdot t-10.224, \\
& \beta_{1}=8904.1 \cdot t^{6}-31042 \cdot t^{5}+38856 \cdot t^{4}-21225 \cdot t^{3}+4992.3 \cdot t^{2}-504.78 \cdot t+9.8932, \\
& \beta_{2}=10306 \cdot t^{6}-30959 \cdot t^{5}+34613 \cdot t^{4}-17132 \cdot t^{3}+3013.6 \cdot t^{2}+165.44 \cdot t-55.019 .
\end{aligned}
$$

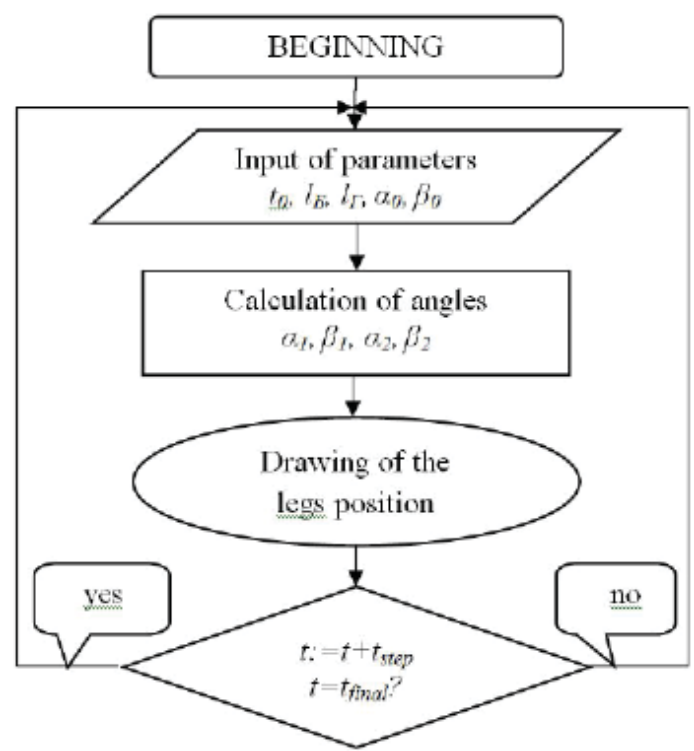

Figure 5: Block diagram of visualization 

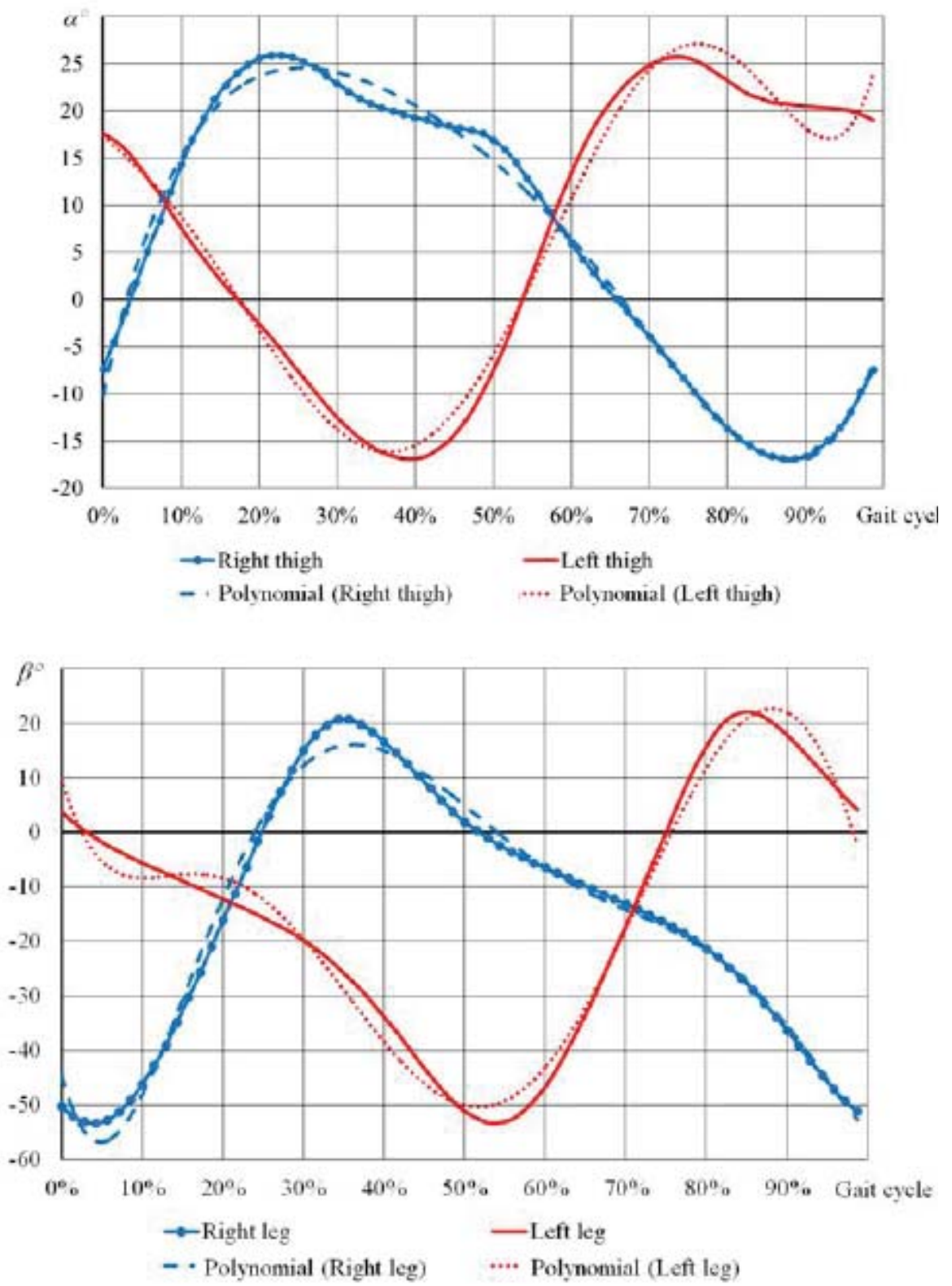

Figure 6: Change of basic angles while walking [according to 9]

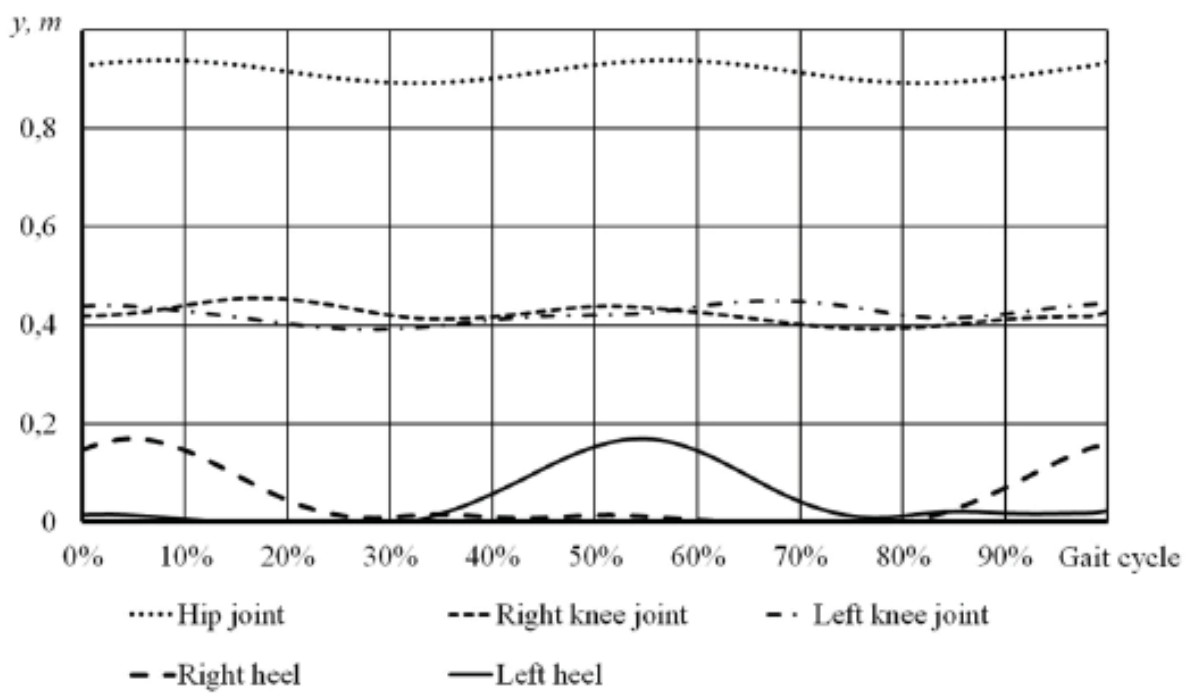

Figure 7: Vertical coordinates of the key points 


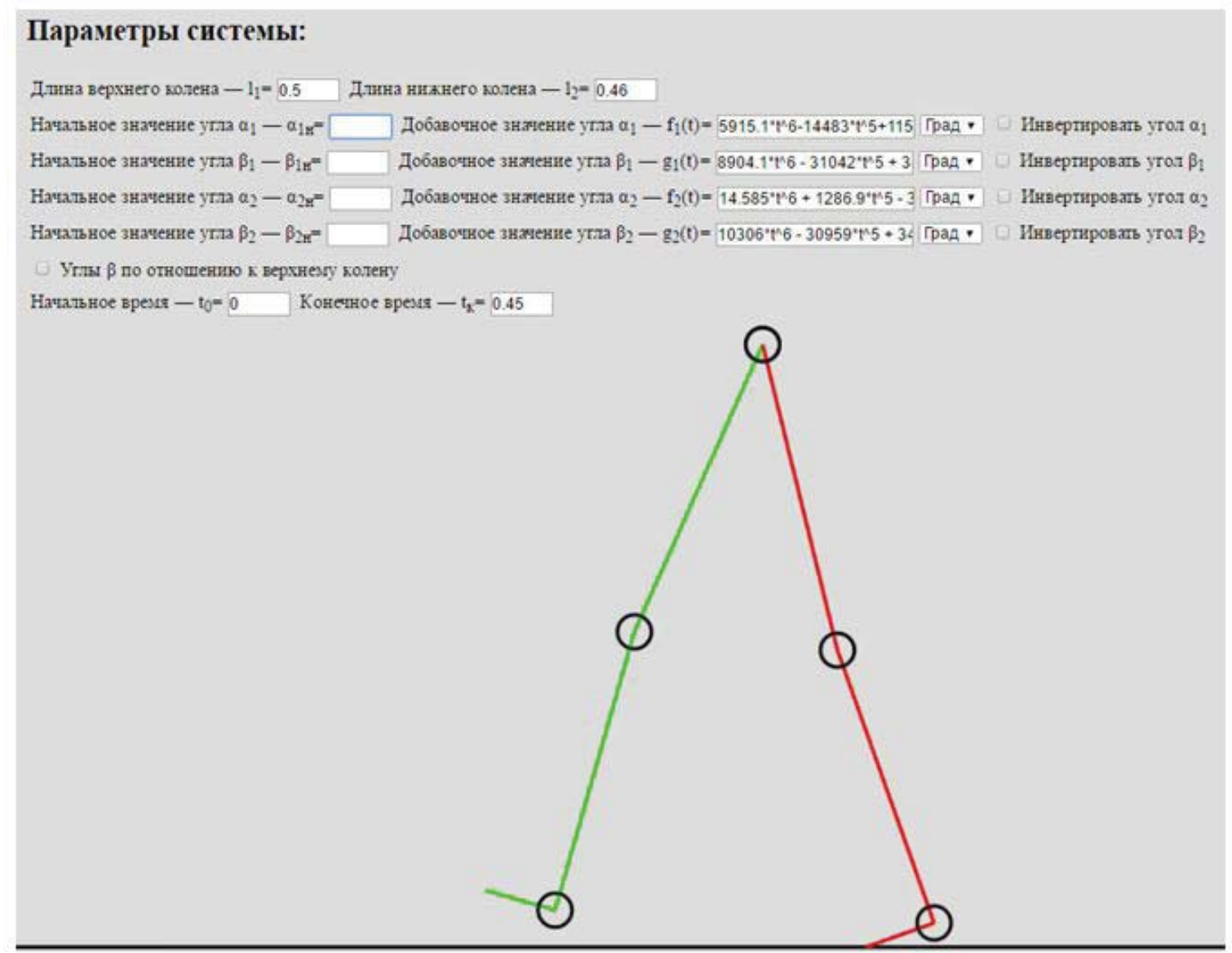

Figure 8: Computer modelling of locomotions

\section{CONCLUSIONS}

1. The calculated mathematical model for regular human walking was developed. The characteristics of the gait necessary for the design of the kinematic model of motion were de-scribed. The laws describing the key points of legs (heel, knee, pelvic joint) were presented.

2. The computer modelling of the gait was done. Based on the calculated mathematical model of the biped gait, we offered an algorithm and developed Web-interface software to implement it. The developed software visualizes the results of the computer modeling of the gait. The dynamic demonstration can be observed here: https://alexanderfadeev.github.io/ legmovement.html

3. A kinematic analysis of the human gait was presented as well as the diagrams showing the change of key leg points of heel, knee and the point of joint of legs and the body in time.

4. Based on the calculated mathematical model, kinematic analysis of locomotions and computer modelling of the gait, the synthesis of the laws of motion for REM system was done.

5. Performed in the given work research can help solve the problem of synthesis of laws of motion while walking and use it for control system of servo drives of a rehabilitation ex-oskeleton for medical purpose.

6. In the future the obtained results can be used to create the dynamic model of biped gait. The model will take into account the reactions of the support, the control moments in the knee and pelvic joints both for the human body and the exoskeleton.

\section{ACKNOWLEDGEMENT}

The work was carried out in the framework of the comprehensive project aimed at setting up the high-tech production «Setting up the high-tech production of a multifunction robotic exoskeleton for medicinal purposes» («REM»), cipher 2017-218-09-1807, approved by the decree of the Government of the Russian Federation № 218 dated April 9, 2010.

\section{REFERENCES}

1. Hugh Herr. (2009) Exoskeletons and orthoses: Classification, design challenges and future directions. Journal of Neuro Engineering and Rehabilitation. 6(21):21. DOI: 10.1186/1743-0003-6-21

2. Vorobiev A.A., Petrukhin A.V., Zasypkina O.A., Krivonozhkina P.S. (2015) Ekzoske-let - novye vozmozhnosti abilitatsii i reabilitatsii (analitichesky obzor) [Exoskeleton is New Possibilities of Habilitation and Rehabilitation (Analytical Survey).] Voprosy re- 
konstruktivnoy i plasticheskoy khirurgii [Problems of Reconstructive and Plastic Surgery]. Vol. 18. No 2 (53). Pp. 51-62.

3. Cristian C. Velandia, Diego A. Tibaduiza, Maribel Anaya Vejar. Proposal of Novel Model for a 2 DOF Exoskeleton for Lower-Limb Rehabilitation // Robotics, MDPI. - 2017, 6 (20). P. 1- 25. doi:10.3390/ robotics6030020https://pdfs.semanticscholar.org/ ab30/9d109fc6e405341f6eba985b68f431c4f348.pdf

4. D. X. Liu, X. Wu, W. Du, C. Wang, C. Chen, and T. Xu, (2017) Deep spatial-temporal model for rehabilitation gait: optimal trajectory generation for knee joint of lower-limb ex-oskeleton. Assembly Automation, vol. 37, no. 3, pp. 369-378. https://doi.org/10.1108/ AA-11-2016-155

5. Sado F, Yap HJ, Ghazilla RAR, Ahmad N (2018) Exoskeleton robot control for syn-chronous walking assistance in repetitive manual handling works based on dual unscented Kalman filter. PLoS ONE 13(7): e0200193. https://doi.org/10.1371/journal. pone.0200193

6. Jianhua Chen, Xihui Mu, Fengpo Du. (2017) Biomechanics analysis of human lower limb during walking for exoskeleton design. Journal of Vibroengineering, Vol. 19, Issue 7, P. 5527-5539. https://doi. org/10.21595/jve.2017.18459 или https://www.jvejournals.com/article/18459

7. Qiming Chen, Hong Cheng, Chunfeng Yue, Rui Huang, and Hongliang Guo. (2018) Dynamic Balance Gait for Walking Assistance Exoskeleton. Applied Bionics and Biome-chanics. Volume 2018, 10 p. Article ID 7847014, https://doi.org/10.1155/2018/7847014

8. Loskutov Yu. V., Kapustin A. V., Klyuzhev K. S., Kudryavtsev A. I., Loskutov M. Yu., Fadeev A. M. (2017) Coputer Simulation of Regular Walking Based on the Kinematic Analysis of Movements and the Synthesis of Exoskeleton Control Algorithms. Vestnik of Volga State University of Technology. Ser.: Radio Engineering and Infocommunication Sys-tems.. No 3 (35). Pp. 47-60. DOI: 10.15350/2306-2819.2017.3.47

9. Winter, David A., Biomechanics and motor control of human movement / David A. Winter. - JOHN WILEY \& SONS, INC., 2009 - 370 p. ISBN 978-0-47039818-0 (cloth)

10. Michael Oluwatosin Ajayi. Modelling and control of actuated lower limb exoskele-tons : a mathematical application using central pattern generators and nonlinear feedback con-trol techniques. General Mathematics [math.GM]. Université Paris-Est, 2016.
English. https://tel.archives-ouvertes.fr/tel-01531927

11. Skvortsov, D.B., Diagnostics of the motion activity with instrumental methods: gait analysis, stabilometry / D.V. Skvortsov. - M.: T.M. Andreeva. 2007. $640 \mathrm{p}$.

12. Kapandzhi, A.I. Nizhnyaya konechnost: Funktsional'naya anatomiya [Lower Limb: Physiological Anatomy] / A.I. Kapandzhi; preface Prof. Thierry Judet; [Translation from French G. Abeleva, E. Kishenevsky]. Moscow: Publishing house «E»2017. 352 p. ISBN 978-5-699-43912-6.

13. Mark L. Latash, Vladimir Zatsiorsky. Biomechanics and Motor Control: Defining Central Concepts. - Elsevier, Academic Press, 2016. - p. 426.

14. T. Koolen, T. D. Boer, J. Rebula, A. Goswami, and J. Pratt. (2012) Capturability-based analysis and control of legged locomotion, part 1: theory and application to three simple gait models. The International Journal of Robotics Research, vol. 31, no. 9, pp. 10941113. https://doi.org/10.1177/0278364912452673

15. J. Pratt, T. Koolen, T. D. Boer et al., (2012) Capturability-based analysis and control of legged locomotion, part 2: application to $\mathrm{m} 2 \mathrm{v} 2$, a lower-body humanoid. The International Journal of Robotics Research, vol. 31, no. 10, pp. 1117-1133. https://doi. org/10.1177/0278364912452762

16. Kolesnikova G.P., Formalsky A.M. (2014) Ob odnom sposobe modelirovaniya pok-hodki cheloveka [About One Method of Human Gait Modeling]. Inzhenernyy zhurnal: nauka i innovatsii [Engineering Journal: Science and Innovations] No 1 (25). P. 11. http://engjournal.ru/catalog/eng/teormech/1181.html. (reference data 10.07.2017).

17. Apaleeva A.M. (2012) Razrabotka algoritma issledovaniya kinematiki dvizheniya cheloveka [Development of the Research Algorithm of Human Motion Kinematics]. Vestnik Amurskogo gosudarstvennogo universiteta. Seriya: Estestvennye i ekonomicheskie nauki [Vestnik of Amur State University. Series: Natural and Economic Sciences]. No 59.Pp. 12-17.

18. The objective assessment of the gait function: clinical recommendations / D.V. Skvortsov and others. - M.: Stroke struggle National Association, Rehabilitation Union of the RF, Russian Association for sport medicine and rehabilitation of disabled people, Interregion-al Public Organization "Union of Neuroanesthesia and neuroresuscitation specialists", 2016. -25 p.

Paper submitted: 21.04.2018.

Paper accepted: 05.12.2018.

This is an open access article distributed under the CC BY-NC-ND 4.0 terms and conditions. 